\title{
A high resolution long travel friction-drive micropositioner with programmable step size
}

\author{
S. H. Chang ${ }^{\text {a) }}$ and S. S. Li \\ Department of Mechanical Engineering, National Taiwan University, Taipei, Taiwan, Republic of China
}

(Received 20 May 1998; accepted for publication 16 February 1999)

\begin{abstract}
A piezoelectric-driven Scott-Russel linear micropositioner utilizing the stick-slip effect of friction to drive a slider is presented. Effects of sawtooth, impulse, and transcendental electrical wave forms on the device performance are studied via numerical simulation and experiment test. The experiment demonstrates that positioning step sizes of $0.05-120 \mu \mathrm{m}$ can be achieved at low input voltages of 2-25 V and essentially with unlimited travel range. (C) 1999 American Institute of Physics.

[S0034-6748(99)00606-1]
\end{abstract}

\section{INTRODUCTION}

The need for precise micropositioning arises in many fields of research and technology, such as in cellular biology, lithography, scanning tunneling microscopy, and scanning probe microscopy. In order to minimize the volume, reduce the cost, and raise the quality of products, the industry is directed into the goal of high precision, high speed, and minimization. In the precision positioning equipment, usually the travel range of these devices is limited by the fine positioning capability. In semiconductor industries, the fabrication process of the $12 \mathrm{in}$. diameter wafer needs microstepping with fine feature size in large travel range. Consequently, developing new generation of positioners to achieve large and fine travel capability is the goal of this article.

In reviewing literatures, the precision positioning mechanisms mainly include the stick-slip effect induced friction drive stage, the clamp-release inchworm-type stage, and the elastic deformation type stage.

In the friction drive mechanisms, the principle of inertial sliding in response to a periodically accelerated input wave form was employed ${ }^{1}$ to design a piezoelectric tube actuating positioning device with long travel range. It provides the resolution of $0.04-0.2 \mu \mathrm{m}$ and could be loaded up to $1 \mathrm{~kg}$ under electrical amplitude of 60-300 V. A similar principle was taken to design the long travel stage with the shear deformation of a piezoelectric plate as the driving force. ${ }^{2}$ The resolution of $10 \mathrm{~nm}$ and the speed of $0.4 \mathrm{~mm} / \mathrm{s}$ with an electrical amplitude of $350 \mathrm{~V}$ were reported. A vertical piezoelectric inertial slider against gravity was developed. ${ }^{3}$ The cycloidal wave forms instead of sawtooth signals were used to activate the motion at a single step of $3 \mathrm{~nm}$. A highresolution two-dimensional (2D) micropositioning device ${ }^{4}$ for scanning probe microscopy utilized piezoelectric element driven by the impulse voltage to achieve the step displacement as small as $10 \mathrm{~nm} / \mathrm{step}$. The device which employed the design of $\mathrm{V}$ grooves in the $X$ and $Y$ directions significantly reduced the crosstalk between directions. It was driven at the

${ }^{a)}$ Electronic mail: shchang@ccms.ntu.edu.tw frequencies between 1 and $4 \mathrm{kHz}$ and at $300 \mathrm{~V}$. A nanostep movement based on the changes of inertia, shock, or clamp force was reported ${ }^{5}$ with results of a single step from 10 to $1000 \mathrm{~nm}$.

The linear piezomotor ${ }^{6}$ made by Burleigh Instruments is the typical design of the inchworm type. A 2D micropositioner $^{7}$ for scanning tunneling microscopy using bimorph piezoelectric ceramic deformation and electrostatic clamping force achieved motion steps from 25 to $40 \mathrm{~nm}$. A simple $X Y \theta_{z}$ walker $^{8}$ used deformation of piezoelectric elements with the aid of clamp release by electromagnetic force. The similar piezomotor ${ }^{9}$ uses five piezoelectric actuators to achieve the $5 \mathrm{~mm}$ travel range with step size from $5 \mathrm{~nm}$ to 3 $\mu \mathrm{m}$. Another piezomotor ${ }^{10}$ uses three piezoelectric actuators to achieve high stiffness of $90 \mathrm{~N} / \mu \mathrm{m}$ output force of $200 \mathrm{~N}$, step resolution of $5 \mathrm{~mm}$, and speed of $6 \mathrm{~mm} / \mathrm{s}$. However, it takes six steps of actuation to achieve one step motion. The complexity of the control to handle the plural piezoelectric actuators becomes its drawback.

The third type is based on the fine expansion of piezoelectric effects and elastic material's deformation. The typical design of this kind is credited to the piezoelectric stage developed by Scire and Teague. ${ }^{11}$ Another design, a bimorph-driven $X-Y-Z$ translation stage ${ }^{12}$ achieved the sensitivity of deformation of $0.3 \mu \mathrm{m} / \mathrm{V}$ and the moving range was $660 \mu \mathrm{m}$. The design of using leaf springs was found in a three-dimensional (3D) microposition mechanism. ${ }^{13}$ ScottRussel linkage was integrated in a linear micropositioner ${ }^{14}$ and achieved resolution of $40 \mathrm{~nm}$ with a travel of greater than $100 \mu \mathrm{m}$. A piezoelectric driven precision $X Y \theta_{z}$ microposition mechanism ${ }^{15}$ achieved the displacement sensitivity of $0.12 \mu \mathrm{m} / \mathrm{V}$ with $10 \mathrm{~nm}$ resolution in the $X$ and $Y$ axes, $4.2 \mu \mathrm{rad} / \mathrm{V}$ with $0.15 \mu \mathrm{rad}$ resolution in the $\theta z$ axis and interaction between axes was within $1.2 \%$.

Force capability is an important specification of actuators. The information can be found in few articles. The force generated by piezoactuators can be up to $9.8,{ }^{1} 15,{ }^{6} 200,{ }^{10}$ and $49 \mathrm{~N} .{ }^{15}$ In general, due to the stiffness, the bimorph piezoactuator provides much less force than multilayer piezoactuator. The inchworm-type and stick-slip-type actuators provide less force than the elastic deformation type. 


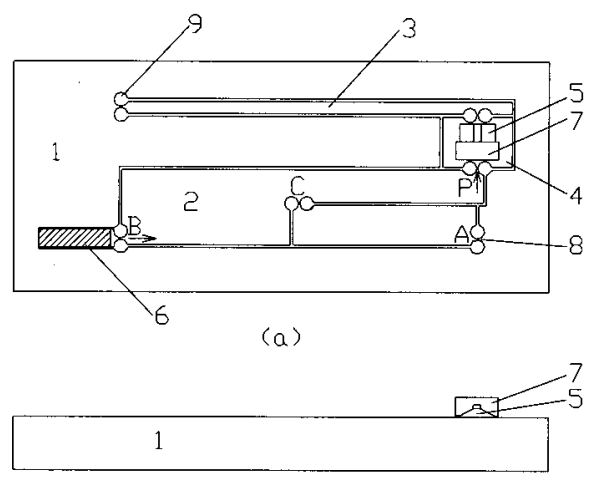

(b)

FIG. 1. A Scott-Russel mechanism.

In viewing the drawbacks of the control complexity in the second type and the short travel range in the third type, the principle of friction drive mechanism is chosen in this design. The advantage of the present design is the use of the linear magnifying mechanism to allow the input voltages ranging from 2 to $25 \mathrm{~V}$ which weighs $3 \%-7 \%$ of other devices. Moreover, the linear magnifying mechanism offers both large and small step size of the micropositioner. The monolithic machined micropositioner by the wire electrodischarge machine integrates functional mechanisms into one piece, simplifies the complicated assembling, and improves the precision of linear motion. A new type of linear precision micropositioner with the results of mathematical simulation and experimental test is presented.

This device provides potential applications to semiconductor equipment, such as the stepper and aligner for optical lithography. The micropositioner also offers scanning tunneling microscopes, scanning probe microscopes, and profile measurement devices for topographies of relatively large areas with high resolution.

\section{PRINCIPLE, DESIGN, AND FABRICATION}

\section{A. Basic mechanism}

The design of the micropositioner is based on the principle of the friction drive to achieve long travel range with fine step motion. The micropositioner as shown in Fig. 1 mainly comprises two parts: the actuation device and a slider. The actuation device includes a multilayer piezoelectric actuator, a linear magnifying mechanism, a parallel guiding mechanism, and a movable platform. The V-shape guide with its surface in direct contact with the slider is to induce stick-slip effect and ensure self-alignment of the slider.

Multilayer piezoelectric ceramic serves as the actuator and has merits of extremely fine displacement sensitivity, fast response, low heat generation, and easy control. By the excellent dynamic response of the piezoelectric ceramic, the tiny displacements in response to input voltage can be obtained. With the layer thickness of approximate $125 \mu \mathrm{m}$, the $5 \times 5 \times 20 \mathrm{~mm}^{3}$ multilayer piezoelectric ceramic is capable to expand $11.5 \mu \mathrm{m}$ and generate the force of $567 \mathrm{~N}$ at $100 \mathrm{~V}$ with the natural frequency of $69 \mathrm{kHz}$. The linear magnifying mechanism employed in this design essentially is a Scott-

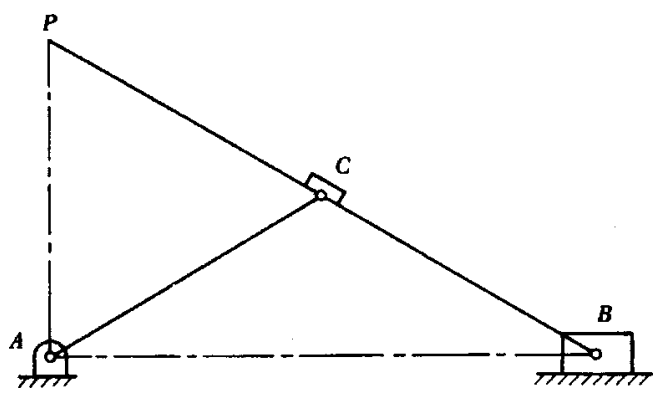

FIG. 2. Schematic representation of the whole micropositioner. 1: the base plate, 2: the linear magnifying mechanism, 3: the parallel guiding mechanism, 4: the movable platform, 5: V-shape guide, 6: the piezoelectric actuator, 7: the slider, 8: one of the flexure hinges, 9: cut-out. The arrows indicate the direction of the motion.

Russel mechanism to enlarge step size of the slider and also reduce input voltage. The principle of Scott-Russel mechanism $^{16}$ is shown in Fig. 2 where $\mathrm{A}$ is the fixed pin joint, $\mathrm{C}$ is a pin connecting links $\overline{\mathrm{AC}}$ and $\overline{\mathrm{BP}}$, and slider $\mathrm{B}$ moves along the horizontal direction. At the conditions of $\overline{\mathrm{AC}}=\overline{\mathrm{BC}}=\overline{\mathrm{CP}}$, point $\mathrm{P}$ will follow an exact vertical straightline motion when slider B moves horizontally.

In Fig. 1 piezoelectric actuator 6 connects, through a flexural hinge, the linear magnifying mechanism 2 which further connects, through other flexural hinges, the movable platform 4. At the opposite end of the movable platform 4, it is connected to, through another flexural hinge, the parallel guiding mechanism 3 which has a relative long link to ensure the linear motion of the platform. The slider 7 rides on the top surface of the V-shape guide 5 that is part of the movable platform. The linear magnifying mechanism, as indicated by B-C-P-A-B in Fig. 1, is a Scott-Russel mechanism corresponding to Fig. 2. The arrowheads indicate the direction of the motion. This configuration of structure ensures compactness and simplicity of the micropositioner. The length of the piezoelectric actuator 6 changes due to the driving voltage between the electrodes of the piezoelectric actuator. This length change pushes or pulls the input terminal, point B, of the linear magnifying mechanism and causes displacement of the output of the mechanism at point $\mathrm{P}$. The parallel guiding mechanism 3 joined to the movable platform is to prevent the platform from rotation and lateral motion. Then the movable platform 4 follows in phase with the output motion at $\mathrm{P}$. The Scott-Russel mechanism is designed to amplify the input displacement so that the travel range of the movable platform is significantly larger than the length changes of the actuator. The slider is placed on the top of the $\mathrm{V}$-shape guide of movable platform with the preload springs. The step motion of the slider can be achieved based on the stick-slip effect between the slider and the movable platform.

A C-language program was written to generate the input voltage wave forms to drive the piezoelectric actuator through a AD/DA card. Different wave forms were generated to drive the piezoelectric actuator and cause different motions of the slider. When the piezoelectric actuator receives the input voltage wave form the AD/DA card, the displacement of the movable platform will be enlarged about threefold through the linear magnifying mechanism. The function of the linear magnifying mechanism is to control the 
range of the step size of the slider. It offers the micropositioner an excellent ability to easily program the step size of the slider at relative low input voltages.

\section{B. Finite element method}

The design criteria is used to achieve the micropositioner with the maximum displacement gain and the minimum lateral deviation. A critical issue in designing such type of mechanism, the stretching and bending of the flexures may occur simultaneously. For the benefits of micropositioner, the stretching should be minimized and the bending should be fully used. The parallel guiding mechanism and movable platform essentially have no stretch due to the relatively long linkages. Links AC and BCP may encounter stretching and bending. The geometry of flexural hinges including the diameter of the cut-out slots and the thickness of hinges and the width of links were carefully selected. The bending compliance is much larger than that of stretching. The bending will dominate the mechanism and links AC and BCP simply rotate about their hinges and are not stretched. For this complex geometry structure with different materials, finite element method (FEM) is most adequate to analyze the performance of the micropositioner. In this study, a well known FEM software ANSYS was utilized to simulate the static and dynamic characteristics of the micropositioner. Due to the uniform thickness of the micropositioner, the twodimensional eight-node structural element was chosen to construct its finite element model. The static analysis results of FEM ensure that the domination of bending of linkage. The maximum stresses occurring in flexure hinges does not exceed the allowable stress and the magnification in the linear magnifying mechanism matches design criteria. The lateral deviation of the parallel guiding mechanism is also minimized to satisfy our demand. The dynamic analysis solved the natural frequencies and vibration modes to ensure the high bandwidth and the linear motion of the platform.

The dimension of the micropositioner was carefully chosen based on the FEM analysis results. The length of $\overline{\mathrm{AB}}$ is $100 \mathrm{~mm}$, the length of $\overline{\mathrm{AP}}$ is $20 \mathrm{~mm}$, the thickness of the micropositioner is $15 \mathrm{~mm}$, the width of the flexural hinge is $0.25 \mathrm{~mm}$, the diameter of cut-out circle at the flexural hinge is $4 \mathrm{~mm}$, and the total dimension is $155 \times 70 \times 15 \mathrm{~mm}^{3}$. The mass of the slider is $2.63 \mathrm{~g}$.

\section{Fabrication}

Fabrication of the micropositioner involves two steps: machining the flexure hinges and linkages from a metal blank, and installing the piezoelectric actuator into the micropositioner.

To make the micropositioner more precise and compact, the first operation is to utilize the wire electrodischarge machine (EDM) to fabricate the flexural hinges and cut-out slots in one piece of metal to form geometric shape. Aluminum 6061-T6 is chosen for the base plate due to its high mechanical strength, low density, and rust proof. The magnifying mechanism, the V-shape guide, and the parallel guiding mechanism are machined monolithically in the base plate.

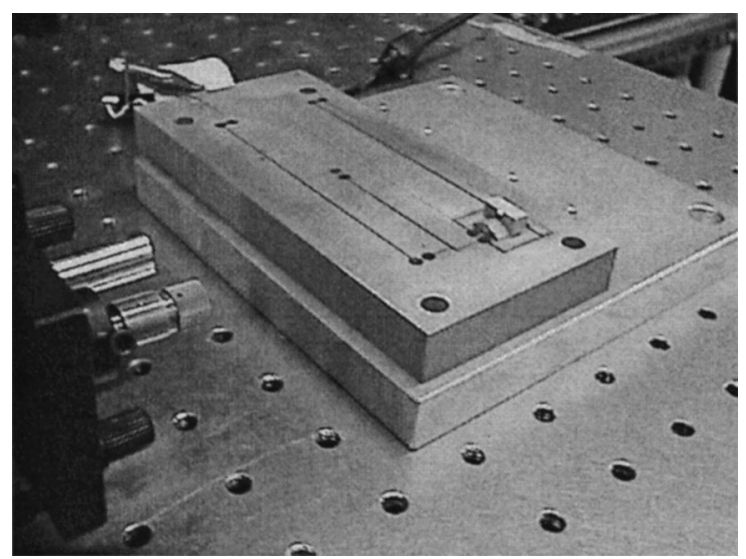

FIG. 3. Photograph of the micropositioner.

The flexural hinges serve as the pivot pins to connect two rigid bodies to provide relative motions. Although it absorbs some strain energy, the flexural hinges eliminate complexity of fasteners and essentially no tolerance exists. The width of the flexural hinges is critical in maintaining the precision motion of the micropositioner. Therefore, a \pm 10 $\mu \mathrm{m}$ tolerance of the thickness and the location of the cut-out circles is chosen. The elastic deflection in flexural hinges obtains high resolution, stable performance, low heat generation, and friction free movement.

The second operation is to assemble the piezoelectric element into the cut-out slots. The two surfaces of the cut-out which fits the element should be ensured as flat and parallel as possible. Nonflatness and nonparallelism are both critical to the undesired angular motion of the micropositioner. Then the multilayer piezoelectric ceramic element was installed into the base plate and then bonded by special structure glue. A photograph of the micropositioner is shown in Fig. 3.

\section{SIMULATION}

A one-dimensional model of the piezoelectric-driven inertial micropositioner is shown in Fig. 4. The equations of motion for the slider resting horizontally on the movable platform are

$$
\begin{aligned}
& (M+m) \frac{d^{2} X}{d t^{2}}=-c \frac{d X}{d t}-k[X-\Delta L(t)]-\mu m g \\
& \times \operatorname{sgn}\left(\frac{d X}{d t}-\frac{d x}{d t}\right), \\
& m \frac{d^{2} x}{d t^{2}}=-\mu m g \times \operatorname{sgn}\left(\frac{d x}{d t}-\frac{d X}{d t}\right),
\end{aligned}
$$

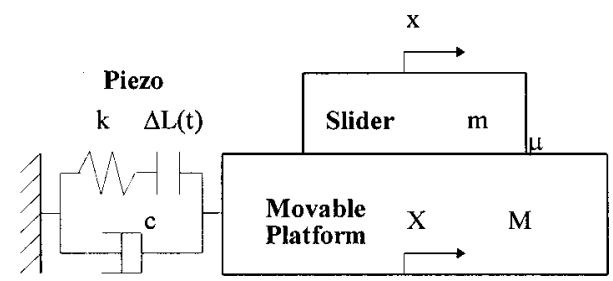

FIG. 4. Simulation model of the piezoelectric-driven inertia micropositioner. 


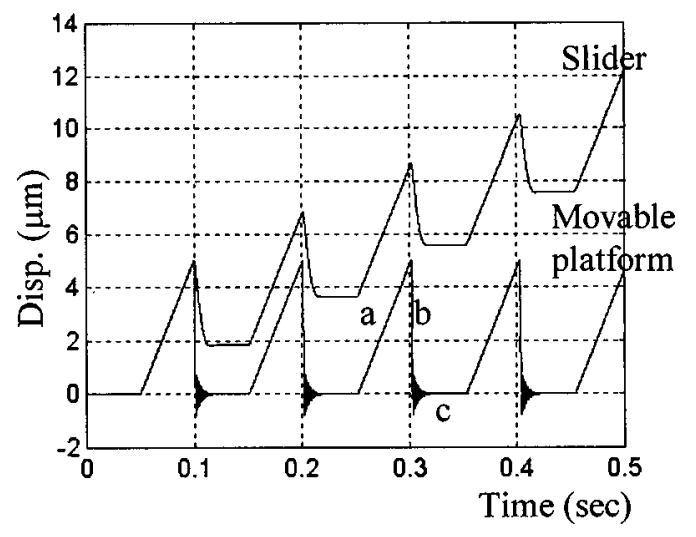

FIG. 5. Calculated results of the displacement of the slider and the movable platform under sawtooth wave form input.

where

if $\left(\frac{d x}{d t}-\frac{d X}{d t}\right)<0, \quad \operatorname{sgn}\left(\frac{d x}{d t}-\frac{d X}{d t}\right)=-1$,

for slipping in the negative direction,

if $\left(\frac{d x}{d t}-\frac{d X}{d t}\right)=0, \quad \operatorname{sgn}\left(\frac{d x}{d t}-\frac{d X}{d t}\right)=0, \quad$ for sticking,

if $\left(\frac{d x}{d t}-\frac{d X}{d t}\right)>0, \quad \operatorname{sgn}\left(\frac{d x}{d t}-\frac{d X}{d t}\right)=1$,

for slipping in the positive direction,

where $M$ is the mass of the movable platform, $m$ the mass of the slider, $X$ the displacement of the movable platform, $x$ the displacement of the slider, $\Delta L(t)$ the extension of the piezoelectric actuator, $k$ the spring constant of the piezoelectric actuator, $c$ the damping coefficient of the piezoelectric actuator, and $\mu$ the coefficient of friction between the slider and the movable platform. In solving the equations of motion, the input wave form is implemented by quantity $\Delta L(t)$.

Apparently, the wave form of input voltage has a strong influence on the performance of the slider. In order to achieve the better design of the micropositioner, the performance of the slider was studied by solving Eqs. (1) and (2). In this study, Runge-Kutta method in software MATLAB was used to solve these nonlinear ordinary differential equations.

Sawtooth, impulse, and transcendental wave forms were used to drive the piezoelectric actuator to provide extension displacement $\Delta L(t)$. With input sawtooth wave form of $\Delta L(t)$, simulation results of the displacement of the slider and the movable platform are shown in Fig. 5. By observing the results, it is clear that the slider sticks to the movable platform at the low slope regions as indicated by a and $\mathrm{c}$ in Fig. 5 and the slip occurs at the high slope regions as indicated by $b$ in Fig. 5. Figure 6 shows results of displacement of the slider with impulse wave form. Obviously, the slider always slips at acute regions of the input wave form.

Simple conclusions can be obtained from results of computer simulation. The stick-slip effect is the main basis in this design. Properly managing the inertial force and the friction force is the key to achieve fine motion of the slider. In

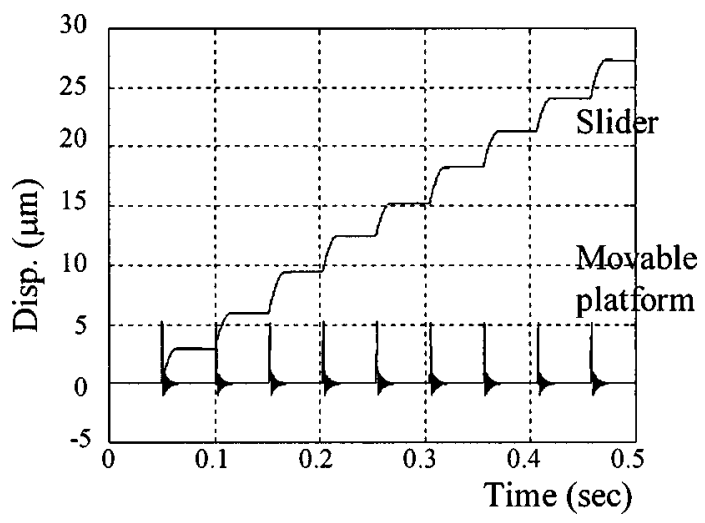

FIG. 6. Calculated results of the displacement of the slider and the movable platform under impulse wave form input.

the low slope region of the input electrical wave forms, the acceleration and inertial force are relatively small in regards to the high slope region of the input wave forms. In this case, the friction force in the contact surface is larger than the inertial force. Thus the slider will stick and stay on the movable platform. In the steep or discontinuous region of the wave form, the acceleration is so large that the inertial force of the slider exceeds the friction force, and hence the slider begins to slip. The single step motion of the slider can be programmed with proper control of the stick and slip conditions. The slider will proceed continuous step motions as long as the periodical voltage wave form is supplied.

When control conditions are changed, the simulation results show that the performance of the micropositioner will be clearly affected. For example, amplitude and frequency of the input wave form, properties of the friction surface, the mass of the slider and the mass of the movable platform are all the main causes to the performance of the micropositioner.

\section{EXPERIMENT AND PEFFORMANCE}

\section{A. Experimental setup}

The experimental setup is shown in Fig. 7. The characteristics of the micropositioner, such as the displacement, angular deviation, and frequency response were measured by a Polytec laser interferometer which has a displacement resolution of $8 \mathrm{~nm}$ and a bandwidth of $50 \mathrm{kHz}$. A photoreflected article was pasted on the appropriate surface of the slider to

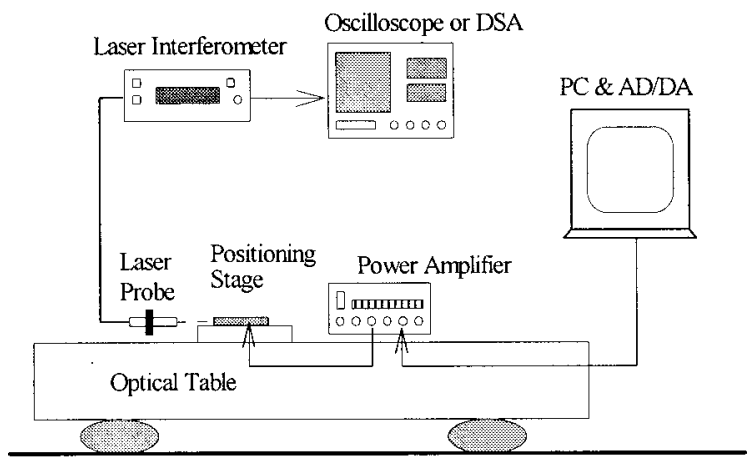

FIG. 7. Experimental setup. 


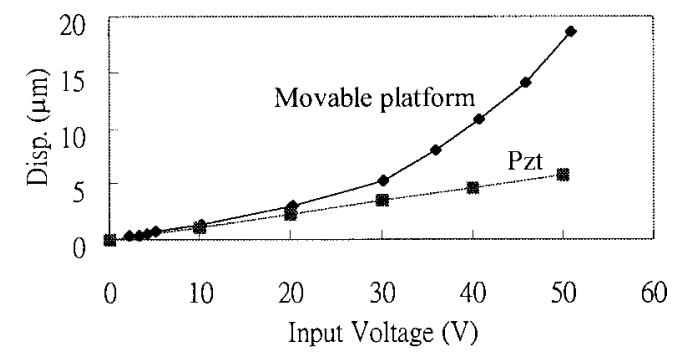

FIG. 8. Dependence of displacement of the movable platform and piezoelectric actuator on input voltage to piezoelectric stack.

aid the laser reflection during the measurement. The desired input wave form is generated by a programmable $\mathrm{AD} / \mathrm{DA}$ card in the personal computer. The output signals generated by the personal computer were amplified by a NP power amplifier to drive the piezoelectric actuator. The Tektronics digital oscilloscope and the dynamic signal analyzer HP35665A were used to capture, display, store, and analyze the experimental signals in the time and frequency domains, respectively. The tested micropositioner was placed on the air-bearing supported optical table to isolate the external vibrations.

\section{B. Characteristics of movable platform}

Displacement sensitivities of the movable platform and the piezoelectric actuator were measured. Figure 8 shows the results and reveals nonlinear characteristics that is simply due to the hysteresis of the piezoelectric actuator. The movable platform of the micropositioner has a displacement of $18.56 \mu \mathrm{m}$ at $50 \mathrm{~V}$. An amplification factor of approximate 3 at $50 \mathrm{~V}$ in the linear magnifying mechanism is found. Because of the loading effect on the piezoelectric element and strain energy stored in the linear magnifying mechanism and in the flexural hinges, the amplification factor of the movable platform is less than that predicted by FEM.

The dynamic responses of the movable platform were measured by the swept sine excitation technique using the dynamic signal analyzer. A typical frequency response function obtained experimentally using the displacement response measured by the laser interferometer is shown in Fig. 9. The first natural frequency of $100 \mathrm{~Hz}$ is then identified. The bandwidth of $100 \mathrm{~Hz}$ is wide enough to operate the

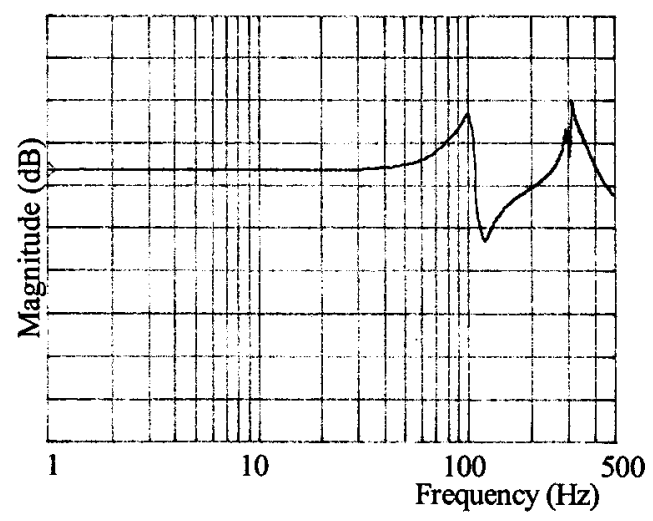

FIG. 9. Frequency response of the movable platform actuated by piezoelectric actuator.

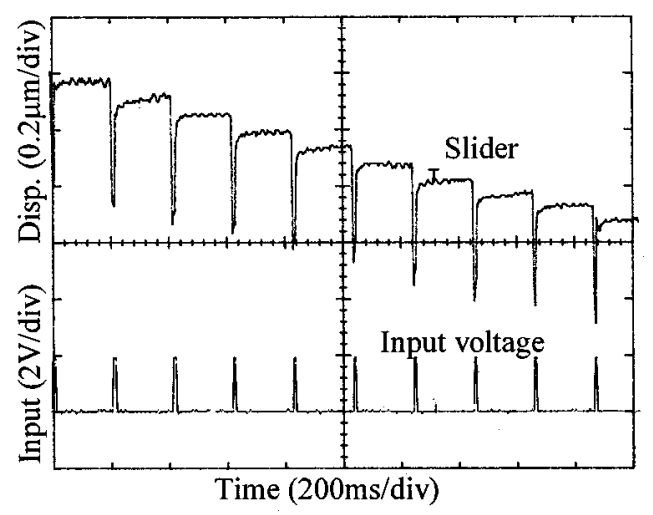

FIG. 10. Actual motion of the slider with $2 \mathrm{~V}$ impulse wave form.

micropositioner in fast response. The mode shape of the micropositioner at $100 \mathrm{~Hz}$ shows the linear movement of the movable platform that matches our design goal.

The displacement sensitivities at various frequencies were also measured. The results are proportional to the amplitude of the frequency response function as shown in Fig. 9. We also conducted the measurements operating near the resonant frequency. In this particular case, the displacement amplitude was so large that the movable platform hit the adjacent structure of the base plate. This problem can be solved if the cut-out slots are made much wider.

\section{Micropositioner performance}

Figure 10 shows results of the measured displacement of the slider under $2 \mathrm{~V}$ impulse wave forms. The displacement signals of the slider show a larger forward motion followed by a smaller backward motion and result a step size of 60 $\mathrm{nm}$. It is clear that the slider sticks to the movable platform then slips at the discontinuous points of the impulse wave form. The fine step size of the slider compared to the relatively large displacement of the movable platform makes the forward and backward motions more noticeable. A step size of $4 \mu \mathrm{m}$ was obtained by increasing the impulse wave form magnitude to $24 \mathrm{~V}$ (Fig. 11). In this case, the step size of the slider is so large that the forward and backward motion is barely noticeable. By controlling the amplitude of the impulse voltages from 2 to $30 \mathrm{~V}$, the step size of the slider can be varied from $50 \mathrm{~nm}$ to $4 \mu \mathrm{m}$.

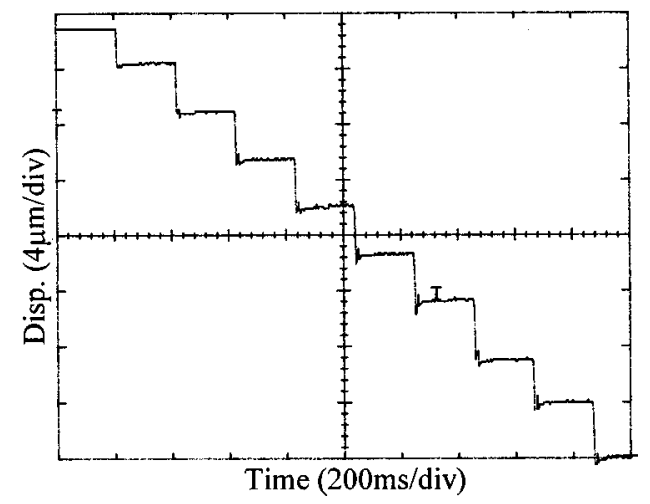

FIG. 11. Actual motion of the slider with $24 \mathrm{~V}$ impulse wave form. 


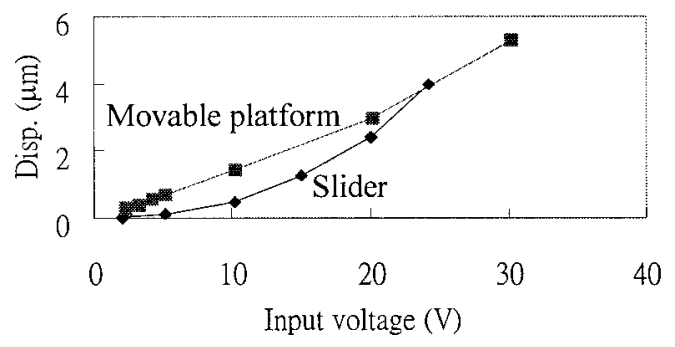

FIG. 12. Comparison of displacement between the slider and the movable platform.

The comparison of displacement between the slider and the movable platform under the impulse excitation is shown in Fig. 12. When input voltage is less than $25 \mathrm{~V}$, the step size of the slider is smaller than the displacement of the movable platform. Besides the slip effect to obtain the step size at the discontinuous points of the input wave form, the slider may receive enough momentum from the friction force and acts as a projectile and exceeds the displacement of the movable platform.

When $20 \mathrm{~V}$ sawtooth wave forms are supplied to the micropositioner, displacement of the slider occurs as is recorded in Fig. 13. The step size of $640 \mathrm{~nm}$ is obtained. It is also equal to the relative motion between the slider and the movable platform as shown in Fig. 14. The relative displacement between these two objects was measured by using differentiation capability of two-probes measurement of the laser interferometer. The slip always occurs in the steep slope region of the input wave form. Comparing experimental results, we concluded that the sawtooth wave form generates the larger step size than using impulse wave form. The step sizes using sawtooth wave form range from $320 \mathrm{~nm}$ to 120 $\mu \mathrm{m}$.

Figure 15 shows the measured displacement of the slider under $15 \mathrm{~V}$ transcendental wave forms. A step size of 480 $\mathrm{nm}$ is found. Again discontinuities in the input wave form generate the relative motion between the slider and the movable platform. The step size of the slider increases with the amplitude of input voltage increases. However, as shown in Fig. 16 it remains constant when the input voltage exceeds $25 \mathrm{~V}$. This indicates that the movable platform is not following the motion of the piezoelectric actuator at high frequency and at high voltage. Consequently, the step size of the slider is limited by the bandwidth of the movable platform. It is

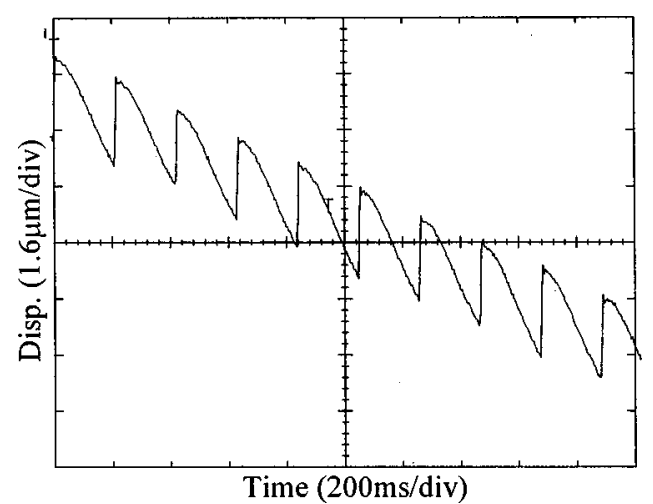

FIG. 13. Actual motion of the slider with $20 \mathrm{~V}$ sawtooth wave form.

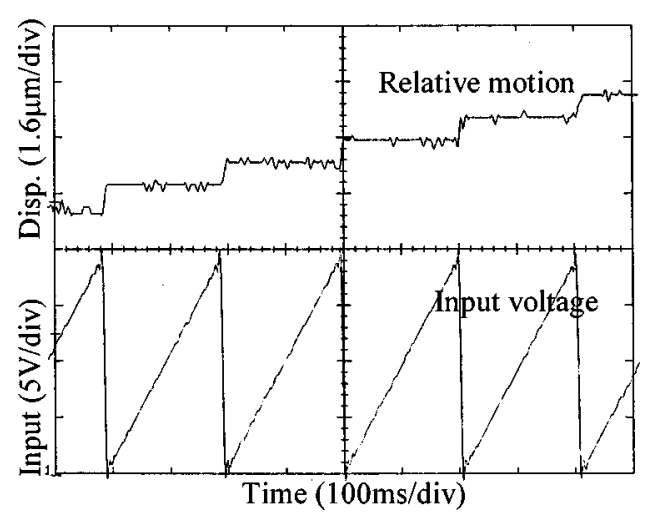

FIG. 14. The relative motion between the slider and the movable platform with $20 \mathrm{~V}$ sawtooth wave form.

concluded that for the larger step size of the micropositioner, the higher bandwidth of the mechanism is required.

\section{CONCLUSION}

A novel micropositioner using multilayer piezoelectric actuator to generate submicrometer-order resolution displacement, a linear magnifying mechanism to enlarge the step size, and integration of stick-slip effect and inertial force to achieve long travel range is developed. The flexural hinges, the linear magnifying mechanism, and the parallel guiding mechanism are machined by a wire electrodischarge machine to ensure the integrity and the precision of the micropositioner. The design utilizes elastic deflection of metal to obtain high resolution, stable performance, low heat generation, and friction free movement. It is simple and compact that all the mechanisms except the slider are machined monolithically in the metal base plate. It simplifies the complicated assembling and improves the precision of linear motion. The mechanical design is accomplished by using a FEM software ANSYS to simulate static and dynamic response of the micropositioner. A further numerical analysis was conducted to solve the nonlinear governing equations. The simulation results predict the performance of the micropositioner under different operating conditions and then provide design criteria.

In experiments, the laser interferometer and dynamic signal analyzer were used to measure static and dynamic response of the micropositioner. We obtain the relation be-

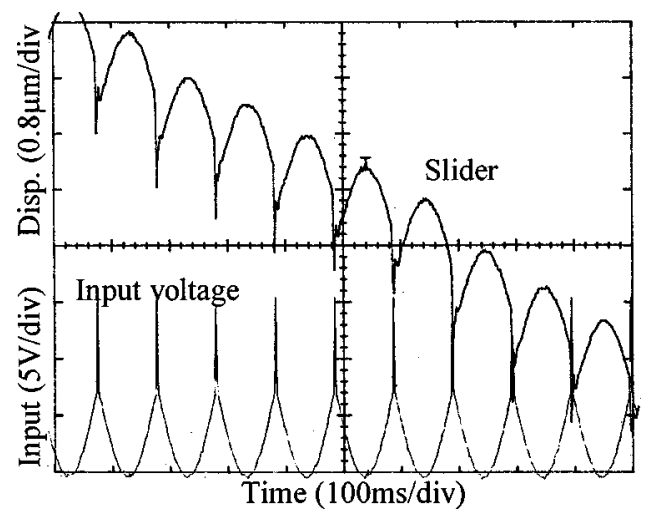

FIG. 15. Actual motion of the slider with $15 \mathrm{~V}$ transcendental wave form. 


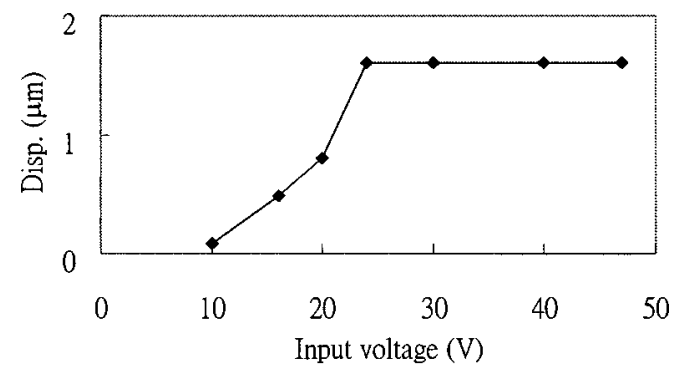

FIG. 16. Actual displacement of the slider with transcendental wave form.

tween the displacement of the movable platform and the input voltage under three electrical wave forms. Different input wave forms applied to the piezoelectric actuator lead to different performance. The impulse, sawtooth, and transcendental wave forms with various amplitudes and frequencies were used to investigate performance of the micropositioner. From simulation and experiment results, the impulse wave form is superior to the other wave forms due to its simplicity and high resolution of displacement. Sawtooth wave forms are capable to generate the largest step sizes. The measured step size of the micropositioner ranges from $50 \mathrm{~nm}$ to $120 \mu \mathrm{m}$ and the speed is up to $2.5 \mathrm{~mm} / \mathrm{s}$.

Comparing with other positioning devices, the present micropositioner is operated at the input voltages of $2-25 \mathrm{~V}$ to minimize the energy consumption and improve the efficiency of the piezoelectric actuator. Moreover, the design of the linear magnifying mechanism allows the change of the step size to be larger, easier, and reliable.

\section{ACKNOWLEDGMENT}

This work was supported by the National Science Council, Taiwan, Republic of China, under Grant No. NSC872215-E-002-016.

${ }^{1}$ D. W. Pohl, Rev. Sci. Instrum. 58, 54 (1987).

${ }^{2} \mathrm{Ph}$. Niedermann, R. Emch, and P. Descouts, Rev. Sci. Instrum. 59, 368 (1988).

${ }^{3}$ Ch. Renner, Ph. Niedermann, A. D. Kent, and O. Fischer, Rev. Sci. Instrum. 61, 965 (1990).

${ }^{4}$ A. R. Smith, S. Gwo, and C. K. Shih, Rev. Sci. Instrum. 65, 3216 (1994).

${ }^{5}$ S. Ya. Tipissev and A. O. Golubok, Tribol. Int. 29, 373 (1996).

${ }^{6}$ W. G. May, Jr., U.S. Patent 3,902,084 (1975).

${ }^{7}$ H. Jonathon Mamin, D. W. Abraham, E. Ganz, and J. Clarke, Rev. Sci. Instrum. 56, 2168 (1985).

${ }^{8}$ V. G. Dudnikov, D. V. Kovalevsky, and A. L. Shabalin, Rev. Sci. Instrum. 62, $2492(1991)$

${ }^{9}$ N. Shimizu, T. Kimura, T. Nakamura, and I. Umebu, J. Vac. Sci. Technol. A 8, 333 (1990).

${ }^{10}$ B. Zhang and Z. Zhu, IEEE/ASME Trans. Mechatronics 2, 22 (1997).

${ }^{11}$ F. E. Scire and E. C. Teague, Rev. Sci. Instrum. 49, 1735 (1978).

${ }^{12}$ J. R. Matey, R. S. Crandall, and B. Brycki, Rev. Sci. Instrum. 58, 567 (1987).

${ }^{13}$ J. Heil, A. Bohm, M. Primke, and P. Wyter, Rev. Sci. Instrum. 67, 307 (1996).

${ }^{14}$ S. H. Chang and B. C. Du, Rev. Sci. Instrum. 69, 1785 (1998).

${ }^{15}$ S. H. Chang, C. K. Tseng, and H. C. Chien, IEEE Trans. Ultrason., Ferroelect., Freq. Control. (to be published).

${ }^{16}$ G. H. Martin, Kinematics and Dynamics of Machines (McGraw-Hill, New York, 1982), p. 52. 